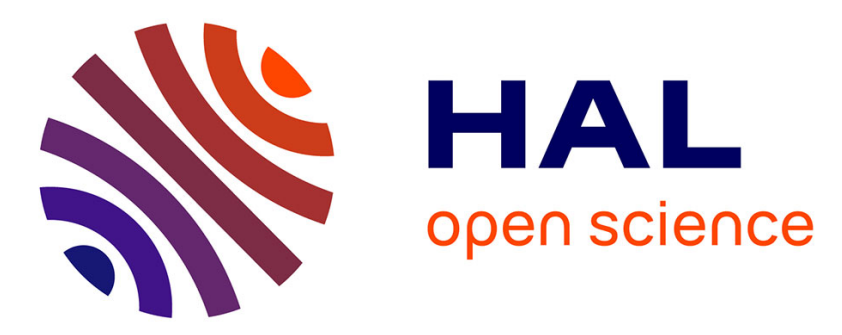

\title{
Conjugate heat transfer with Large Eddy Simulation for gas turbine components
}

Florent Duchaine, Simon Mendez, Franck Nicoud, Alban Corpron, Vincent Moureau, Thierry Poinsot

\section{To cite this version:}

Florent Duchaine, Simon Mendez, Franck Nicoud, Alban Corpron, Vincent Moureau, et al.. Conjugate heat transfer with Large Eddy Simulation for gas turbine components. Comptes rendus de l'Académie des sciences. Série IIb, Mécanique, 2009, 337 (6-7), pp.550-561. 10.1016/j.crme.2009.06.005 . hal00803816

\section{HAL Id: hal-00803816 https://hal.science/hal-00803816}

Submitted on 22 Mar 2013

HAL is a multi-disciplinary open access archive for the deposit and dissemination of scientific research documents, whether they are published or not. The documents may come from teaching and research institutions in France or abroad, or from public or private research centers.
L'archive ouverte pluridisciplinaire HAL, est destinée au dépôt et à la diffusion de documents scientifiques de niveau recherche, publiés ou non, émanant des établissements d'enseignement et de recherche français ou étrangers, des laboratoires publics ou privés. 


\title{
Conjugate Heat Transfer with Large Eddy Simulation. Application to Gas Turbine Components.
}

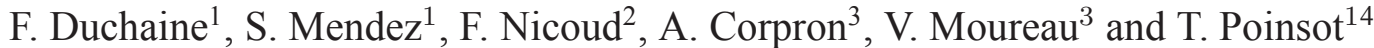

\begin{abstract}
Conjugate heat transfer is a key issue in combustion: the interaction of reacting flows and hot gases with colder walls is actually a main design constraint in gas turbines. For example, multiperforated plates commonly used in combustion chambers to cool walls must be able to sustain the high fluxes produced in the chamber. After combustion, the interaction of the hot burnt gases with the high pressure stator and the first turbine blades conditions the temperature and pressure levels reached in the combustor, and therefore the engine efficiency.

Conjugate heat transfer is a difficult field and most existing tools are developped for chained (rather than coupled), steady (rather than transient) phenomena: the fluid flow is computed using a RANS (Reynolds Averaged Navier-Stokes) solver. During this work, a fully parallel environnement for conjugate heat transfer has been developed and applied to two configurations of interest for the design of combustion devices. The numerical tool is based on a reactive LES (Large Eddy Simulations) code and a solid conduction solver that can exchange data via a supervisor.

An unsteady wall/flame interaction is used to assess the the precision and the order of the coupled solutions depending on the coupling frequency. It is shown that the temperature and the flux across the wall are well reproduced when the codes are coupled with a time scale of the order of the smallest time scale. An experimental film-cooled turbine vane is studied in order to reach a steady state. The solutions from the conjugate analyses and an adiabatic wall convection are compared to experimental results. Concerning pressure profiles, both simulations show a good agreement with experimental results. Due to thermal conduction in the blade, conjugate results has a lower mean intrado temperature than adiabatic simulation and reproduce the experimental cooling efficiency quite well.
\end{abstract}

\section{INTRODUCTION}

Conjugate heat transfer is a key issue in combustion [1,2]: the interaction of hot gases and reacting flows with colder walls is a key phenomenon in all chambers and is actually a main design constraint in gas turbines. For example, multi-perforated plates are commonly used in gas turbines combustion chambers to cool walls and they must be able to sustain the high fluxes produced in the chamber. After combustion, the interaction of the hot burnt gases with the high pressure stator and the first turbine blades conditions the temperature and pressure levels reached in the combustor, and therefore the engine efficiency.

Conjugate heat transfer is a difficult field and most existing tools are developed for chained (rather than coupled), steady (rather than transient) phenomena: the fluid flow is brought to convergence using a RANS (Reynolds Averaged Navier-Stokes) solver for a given set of skin temperatures $[3,4,5]$. The heat fluxes predicted by the RANS solver are then transferred to a heat transfer solver which produces a new set of skin temperatures. A few iterations are generally sufficient to reach convergence. There are circumstances however where this chaining method must be replaced by a full coupling approach. Flames interacting with walls for example, may require a simultaneous resolution of the temperature within the solid and around it. More generally, the introduction of LES to replace RANS leads to full coupling since LES provides the unsteady evolution of all flow variables.

Fully coupled conjugate heat transfer requires to take into account multiple questions. Among them, two issues were considered for the present work:

- The time scales of the flow and of the solid are generally very different. In a gas turbine, a blade submitted to the flow exiting from a combustion chamber has a thermal characteristic time scale of the order of a few seconds while the flow-through time along the blade is less than $1 \mathrm{~ms}$. As a consequence, the frequency of the exchanges between the codes is critical for the precision, stability and restitution time of the computations.

\footnotetext{
${ }^{1}$ CERFACS, CFD team, 42 Av Coriolis, 31057 Toulouse, France

${ }^{2}$ Un. Montpellier II

${ }^{3}$ TURBOMECA, Bordes, France

${ }^{4}$ IMF Toulouse, INP de Toulouse and CNRS, 31400 Toulouse, France
} 
- Coupling the two phenomena must be performed on massively parallel machines where the codes must be not only coupled but synchronized to exploit the power of the machines.

During this work, these two issues have been studied using two examples of conjugate heat transfer: a flame interacting with a wall in section II and a blade submitted to a flow of hot gases in section III. Both problems have considerable impact on the design of combustion devices. Section I deals with the codes used to this studies as well as the coupling strategies.

\section{SOLVERS AND COUPLING STRATEGIES}

The AVBP code is used for the fluid [6,7]. It solves the compressible reacting Navier-Stokes equations with a third-order scheme for spatial differencing and a Runge Kutta time advancement $[8,9]$. Boundary conditions are handled with the NSCBC formulation [10,9].

For the resolution of the heat transfer equation within solids, a simplified version of AVBP, called AVTP, was developed. It uses the same data structure as AVBP. It is coupled to AVBP using a software called PALM [11]. For all present examples, the skin meshes are the same for the fluid and the solid so that no interpolation error is introduced at this level.

The coupling strategy between AVBP and AVTP depends on the objectives of the simulation and is characterized by two issues:

- Synchronization in physical time: the physical time computed by the two codes between two information exchanges may be the same or not. ${ }^{5}$ We will impose that between two coupling events, the flow is advanced in time of a quantity $\alpha_{f} \tau_{f}$ where $\tau_{f}$ is a flow characteristic time. Simultaneously, the solid is advanced of a time $\alpha_{s} \tau_{s}$ where $\tau_{s}$ is a characteristic time for heat propagation through the solid. Two limit cases are of interest: (1) $\alpha_{s}=\alpha_{f}$ ensures that both solid and fluid converge to steady state at the same rate (the two domains are then not synchronized in physical time) and (2) $\alpha_{f} \tau_{f}=\alpha_{s} \tau_{s}$ ensures that the two solvers are synchronized in physical time.

- Synchronization in CPU time: on a parallel machine, codes for the fluid and for the structure may be run together or sequentially. An interesting question controlled by the execution mode is the information exchange. Fig. 1 shows how heat fluxes and temperature are exchanged in a mode called SCS (Sequential Coupling Strategy) where the fluid solver after run $n$ (physical duration $\alpha_{f} \tau_{f}$ ) provides fluxes to the solid solver which then starts and gives temperatures $T_{n}$ (physical duration $\alpha_{s} \tau_{s}$ ). In SCS mode, the codes are loaded into the parallel machine sequentially and each solver use all available processors $(N)$. Another solution is Parallel Coupling Strategy (PCS) where both solvers run together using information obtained from the other solver at the previous coupling iteration (Fig. 1). In this case, the two solvers must share the $P=P_{s}+P_{f}$ processors. The $P_{s}$ and $P_{f}$ processors dedicated to the solid and the fluid respectively must be such that:

$$
\frac{P_{f}}{P}=\frac{1}{1+T_{s} / T_{f}}
$$

where $T_{s}$ and $T_{f}$ are the execution times of the solid and fluid solvers respectively (on one processor). $T_{s}$ and $T_{f}$ depend on $\alpha_{s} \tau_{s}$ and $\alpha_{f} \tau_{f}$. Perfect scaling for both solvers is assumed here.

Note that both SCS and PCS questions are linked to the way information (heat fluxes and wall temperatures) are exchanged and to the implementation on parallel machines but are independent of the synchronization in physical time: PCS or SCS can be used for steady or unsteady computations. This paper focuses on the PCS strategy.

Finally, this work explores the simplest coupling method where the fluid solver provides heat fluxes to the solid solver while the solid solver sends skin temperatures back to the fluid code. More sophisticated methods may be used for precision and stability $[12,13]$ but the present one was found sufficient for the two test cases described below.

\footnotetext{
${ }^{5}$ For example, when a steady state solution is sought (i.e. to be used as initial condition for an unsteady computation), physical times for both solvers can differ.
} 


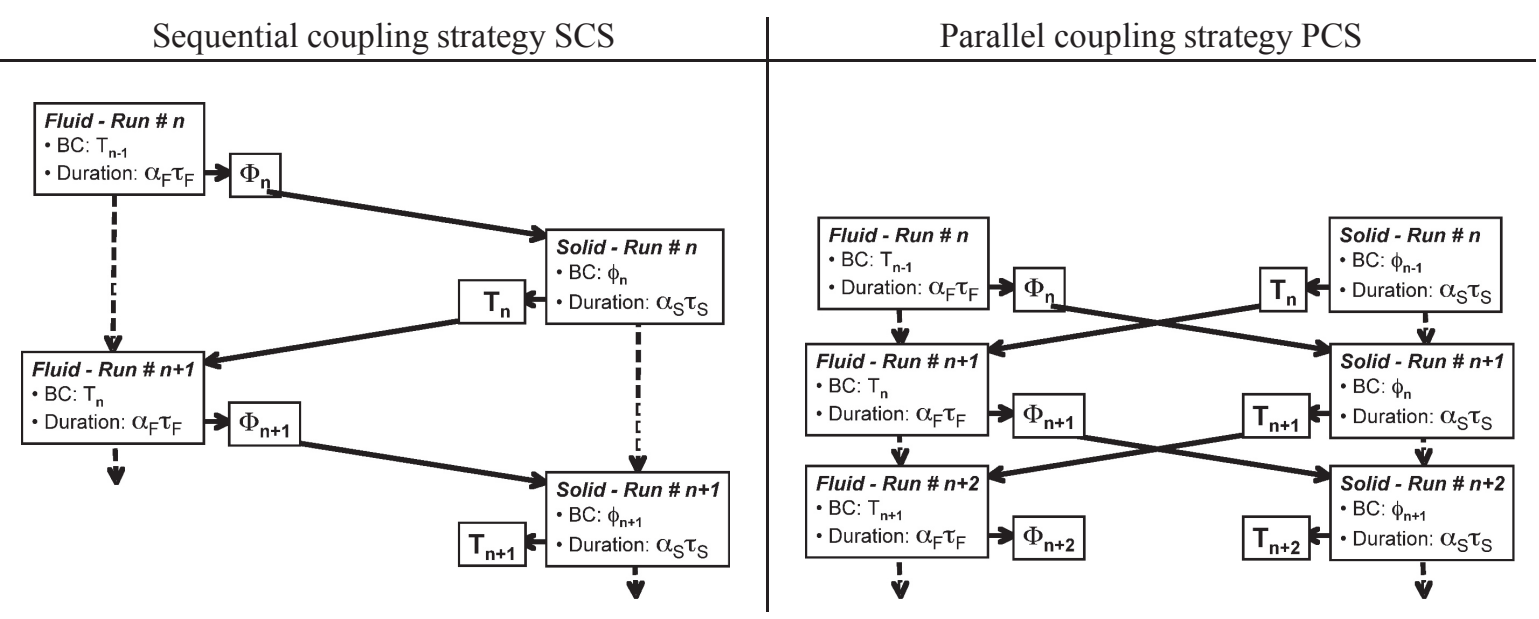

Figure 1 : Main types of coupling strategies.

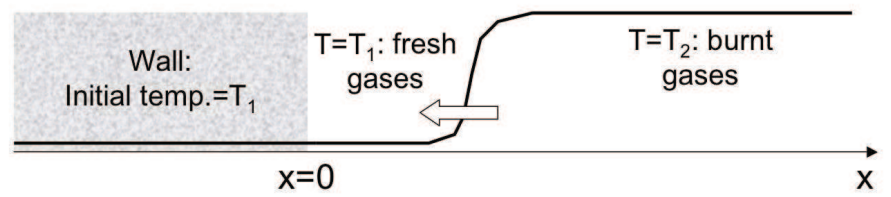

Figure 2 : Interaction between wall and premixed flame. Solid line: initial temperature profile.

\section{FLAME/WALL INTERACTION (FWI)}

The interaction between flames and walls controls combustion, pollution and wall heat fluxes in a significant manner $[10,14,15]$. It also determines the wall temperature and its life time. In most combustion devices, burnt gases reach temperatures between 1500 and $2500 \mathrm{~K}$ while walls temperatures remain between 400 and $850 \mathrm{~K}$ because of cooling. The temperature decrease from burnt gases levels to wall levels occurs in a near-wall layer which is less than $1 \mathrm{~mm}$ thick, creating large temperature gradients.

Studying the interaction between flames and walls is difficult from an experimental point of view because all interesting phenomena occur in a thin zone near the wall: in most cases, the only measurable quantity is the unsteady heat flux through the wall. Moreover, flames approaching walls are dominated by transient effects: they usually do not 'touch' walls and quench a few micrometers away from the cold wall because the low wall temperature inhibits chemical reactions. At the same time, the large near-wall temperature gradients lead to very high wall heat fluxes. These fluxes are maintained for short durations and their characterization is also a difficult task in experiments $[16,17]$.

The present study focuses on the interaction between a laminar flame and a wall (Fig. 2). Except for a few studies using integral methods within the solid [18] or catalytic walls [19, 20], most studies dedicated to FWI were performed assuming an inert wall at constant wall temperature. Here we will revisit the assumption of isothermicity of the wall during the interaction.

\section{II.1 The INFINITELY FAST FLAME (IFF) LIMIT}

Flame front thicknesses $\left(\delta_{L}^{o}\right)$ are less than $1 \mathrm{~mm}$ and laminar flame speeds $\left(s_{L}^{o}\right)$ are of the order of $1 \mathrm{~m} / \mathrm{s}$. Walls are usually made of metal or ceramics and their characteristic time scale $\tau_{s}=L^{2} / D_{s}$ (where $L$ is the wall thickness and $D_{s}$ the wall diffusivity) is much longer than the flame characteristic time $\tau=\delta_{L}^{o} / s_{L}^{o}$. An interesting simplification of this observation is the 'Infinitely Fast Flame' limit (IFF) in which the time scale of the flame is assumed to be zero compared to the solid time. In this case, the FWI limit can be replaced by the simpler case of a semi-infinite solid at temperature $T_{1}$ getting instantaneously in touch with a semi-infinite fluid at a constant temperature $T_{2}$ where $T_{2}$ is the adiabatic flame temperature (Fig. 3). The propagation time of the flame towards the wall is neglected. 


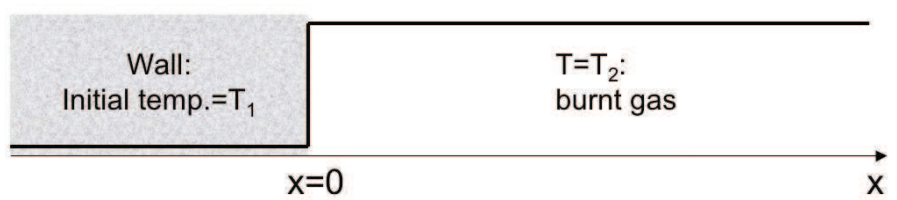

Figure 3 : The IFF (infinitely fast flame) limit. Solid line: initial temperature profile.

\begin{tabular}{ccccccccc} 
& $\begin{array}{c}\text { Initial } \\
\text { temperature }\end{array}$ & $\begin{array}{c}\text { Thermal } \\
\text { diffusivity }\end{array}$ & $\begin{array}{c}\text { Thermal } \\
\text { effusivity }\end{array}$ & $\begin{array}{c}\text { Thermal } \\
\text { conductivity }\end{array}$ & $\begin{array}{c}\text { Heat } \\
\text { capacity }\end{array}$ & Density & $\begin{array}{c}\text { Mesh } \\
\text { size }\end{array}$ & $\begin{array}{c}\text { Fourier } \\
\text { time step }\end{array}$ \\
\hline Solid & 650 & $3.3810^{-6}$ & 7058.17 & 12.97 & 460 & 8350 & $410^{-6}$ & $2.3710^{-6}$ \\
Fluid & 660 & $2.5310^{-5}$ & 5.52 & 0.028 & 1162.2 & 0.947 & $410^{-6}$ & $3.1610^{-7}$
\end{tabular}

Table 1 : Fluid and solid characteristics for IFF test case (SI units). The Fourier time step corresponds to the stability limit for explicit schemes $\Delta t^{D}=\Delta x^{2} /\left(2 D_{t h}\right)$.

The IFF problem is a classical heat transfer problem and has an analytical solution which can be written as:

$$
\begin{gathered}
T(x, t)=T_{1}+b \frac{T_{2}-T_{1}}{b+b_{s}} \operatorname{erfc}\left(-\frac{x}{2 \sqrt{D_{s} t}}\right) \quad \text { for } \quad x<0 \\
T(x, t)=T_{2}-b_{s} \frac{T_{2}-T_{1}}{b+b_{s}} \operatorname{erfc}\left(\frac{x}{2 \sqrt{D t}}\right) \text { for } \quad x>0
\end{gathered}
$$

where $b=\sqrt{\lambda \rho C_{p}}$ is the effusivity of the burnt gases, $b_{s}=\sqrt{\lambda_{s} \rho_{s} C_{p s}}$ the effusivity of the wall and $D$ the burnt gases diffusivity. $D_{s}$ and $D$ are assumed to be constant in the solid and fluid parts. The temperature of the wall at $x=0$ is constant and the heat flux $\Phi$ decreases like $1 / \sqrt{t}$ :

$$
T(x=0, t)=\frac{b T_{2}+b_{s} T_{1}}{b+b_{s}} \quad \text { and } \quad \Phi(x=0, t)=\frac{T_{2}-T_{1}}{b+b_{s}} \frac{b b_{s}}{\sqrt{\pi t}}
$$

This IFF limit is useful to understand FWI limits. It was also used as a test case of the coupled codes to check the accuracy of coupling strategies (next section).

\section{II.2 THE IFF LIMIT AS A TEST CASE FOR UNSTEADY FLUID / HEAT TRANSFER COUPLING}

A central question for SCS or PCS methods is the coupling frequency between the two solvers especially when they have very different characteristic times. Since the IFF has an analytical solution, it was first used as a test case for PCS methods. The test case corresponds to a wall at $650 \mathrm{~K}$ in contact at $t=0$ with a fluid at $660 \mathrm{~K}$. Compared to a wall/flame interaction, this small temperature difference is chosen in order to keep constant values for $D, \lambda$ and $C p$. Table 1 summarizes the properties of the solid and the fluid and indicates mesh size $\Delta x$ and maximum time steps $\Delta t^{D}$ for diffusion (the only important ones here since the flow does not move).

The most interesting part of this problem is the initial phase when fluxes are large and coupling difficult. During this phase, the solid and the fluid can be considered as infinite and there is no proper length or time scale to evaluate $\tau_{f}$ or $\tau_{s}$ in Fig. 1. The only useful scale is the grid mesh and the associated time scale for explicit algorithm stability. Therefore we chose to take $\tau_{f}=\Delta t_{f}^{D}$ and $\tau_{s}=\Delta t_{s}^{D}$ (Note that the fluid solver is limited by an acoustic time step smaller than $\Delta t_{f}^{D}$ ). The strategy used for this test is the PCS method (Fig. 1) for unsteady cases which requires $\alpha_{f} \tau_{f}=\alpha_{s} \tau_{s}$. The $\alpha_{f}$ parameter defines the time interval between two coupling events normalized by the fluid characteristic time. Values of $\alpha_{f}$ ranging from 0.131 to 65.5 were tested for this problem and Fig. 4 shows how the errors on maximum wall temperature and the wall heat flux change when $\alpha_{f}$ changes. The IFF solution 4 is used as the reference solution. Using values of $\alpha_{f}$ larger than unity leads to relative errors which can be significant and to strong oscillations on the temperature and flux. As expected a full coupling of fluid and solid for this problem requires to use values of $\alpha_{f}$ of order unity which means to couple the codes on a time scale which is of the order of the smallest time scale (here the flow time scale). 

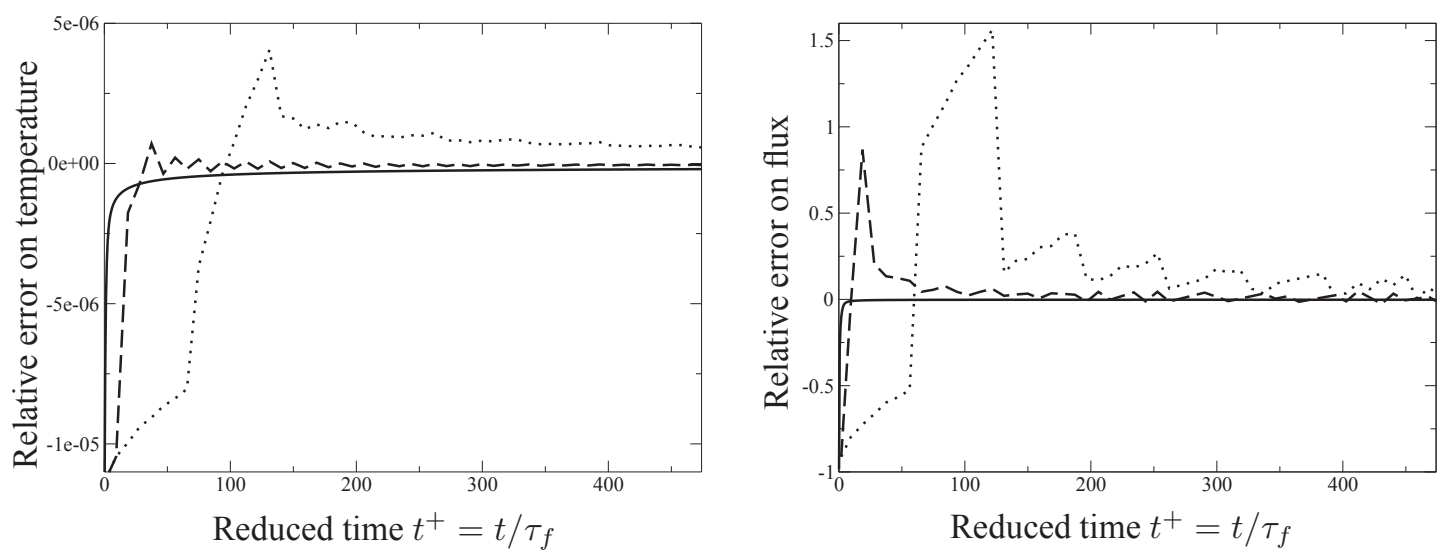

Figure 4 : Tests of the PCS method for the IFF case of Fig. 3. Effects of the coupling period between two events (measured by $\alpha_{s}$ ). Left: relative error on wall temperature at $x=0$, right: relative error on wall flux at $x=0$. Solid line: $\alpha_{f}=0.131$, dashed: $\alpha_{f}=13.1$, dots: $\alpha_{f}=65.5$.

\begin{tabular}{ccccccccc} 
& $\begin{array}{c}\text { Initial } \\
\text { temperature }\end{array}$ & $\begin{array}{c}\text { Thermal } \\
\text { diffusivity }\end{array}$ & $\begin{array}{c}\text { Thermal } \\
\text { effusivity }\end{array}$ & $\begin{array}{c}\text { Thermal } \\
\text { conductivity }\end{array}$ & $\begin{array}{c}\text { Heat } \\
\text { capacity }\end{array}$ & $\begin{array}{c}\text { Density } \\
\text { sesh } \\
\text { size }\end{array}$ & $\begin{array}{c}\text { Time } \\
\text { scale }\end{array}$ \\
\hline Solid & 650 & $3.3810^{-6}$ & 7058.17 & 12.97 & 460 & 8350 & $210^{-6}$ & 0.3 \\
Fresh gases & 650 & $4.1410^{-5}$ & 7.35 & 0.047 & 1168.9 & 0.977 & $410^{-6}$ & $30.4510^{-6}$ \\
Hot gases & 2300 & $3.7210^{-4}$ & 7.28 & 0.140 & 1441.6 & 0.262 & $410^{-6}$ & $30.4510^{-6}$
\end{tabular}

Table 2 : Fluid and solid characteristics for flame/wall interaction test case (SI units). The characteristic time $\tau_{s}$ for the solid is based on its thickness and heat diffusivity. The characteristic time scale for the fluid $\tau_{f}$ is based on the flame speed and thickness.

\section{II.3 FLAME/WALL INTERACTION RESULTS}

This section presents results obtained for a fully coupled FWI and compares them to the IFF limit. The parameters used for the simulation (Table 2) correspond to a methane/air flame at an equivalence ratio of 0.8 , propagating in fresh gases at a temperature $T_{1}$ of $650 \mathrm{~K}$ at a laminar speed $s_{L}^{o}=1.128 \mathrm{~m} / \mathrm{s}$. The adiabatic flame temperature is $T_{2}=2300 \mathrm{~K}$. The wall is initially at $T_{1}=650 \mathrm{~K}$ too. The maximum time step corresponds to the Fourier stability criterion for the solid and to the CFL stability criterion for the fluid. These time steps are respectively $\Delta t_{s}^{F}=0.59 \mu \mathrm{s}$ and $\Delta t_{f}^{C F L}=0.0023 \mu \mathrm{s}$. For this fully coupled problem, the only free parameter is $\alpha_{f}$. The period between two coupling events $\left(\alpha_{f} \tau_{f}=\alpha_{s} \tau_{s}\right)$ determines the number of iterations performed by the gas solver during this time : $N_{i t}=\alpha_{f} \tau_{f} / \Delta t_{f}^{C F L}$. As the cost of computing heat transfer in the solid for this problem is actually negligible, no attempt was made to optimize the computation. The effect of mesh resolution in the gases was also checked and found to be negligible: for most runs, 500 mesh points are used in the gases with a mesh size of $410^{-6} \mathrm{~mm}$.

The coupling parameters for the presented case correspond to a PCS simulation with $\alpha_{f}=7.510^{-3}$ leading to $N_{i t}=100$ in the gases accompanied by one iteration in the solid where $\alpha_{s}=7.610^{-7}$ (Fig. 5). Fig. 6 displays the wall scaled temperature at the fluid and solid interface $T^{*}=\left(T(x=0)-T_{1}\right) /\left(T_{2}-T_{1}\right)$ and the reduced maximum heat flux $\Phi /\left(\rho C_{p} s_{L}^{o}\left(T_{2}-T_{1}\right)\right.$ through the wall versus time. The flame quenches at time $s_{L}^{o} t / \delta_{L}^{o}=9$, where the flux is maximum. Fig. 6 also displays the prediction of the IFF limit (Eq. 4). Except in the first instants of the interaction, when the flame is still active, the IFF limit matches the simulation results extremely well, both in terms of fluxes and wall temperature. The IFF solution can not predict the maximum heat flux because it leads to an infinite flux at the initial time. However, as soon as the flame is quenched, it gives a very good evaluation of the wall heat flux. Note that the wall temperature increases by a small amount during this interaction between an isolated flame and the wall $\left(T^{*} \approx 10^{-3}\right.$ on Fig. 6). In more realistic cases, flame will flop and hit walls at high frequency which could lead to cumulative effects and therefore to higher wall temperature and fatigue.

Fig. 7 shows how the wall temperature at $x=0$ changes when the effusivity of the solid varies. For the IFF limit, this temperature is given by Eq. 4 and for the simulation, it is the temperature reached asymptotically for long times. The agreement is excellent and confirms that the IFF limit correctly predicts the long-term evolution in this FWI problem. It also shows that the coupled simulation works correctly. Note however, 


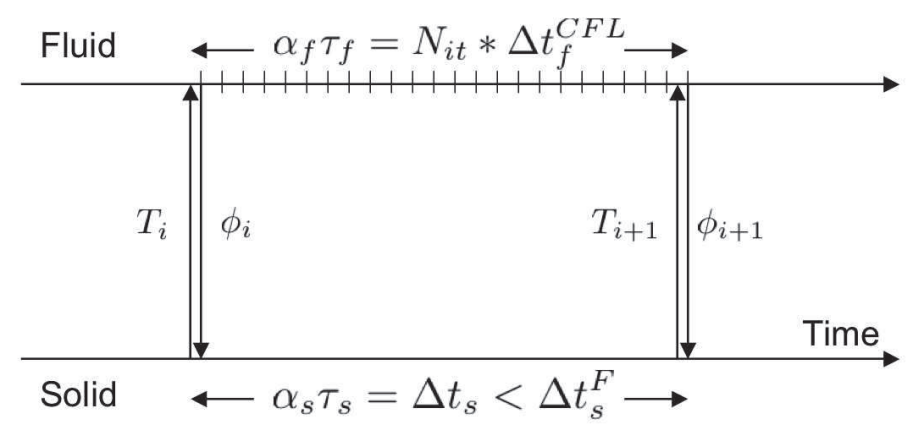

Figure 5 : Parallel coupling strategy for the flame/wall interaction with $\alpha_{f}=7.510^{-3}$.

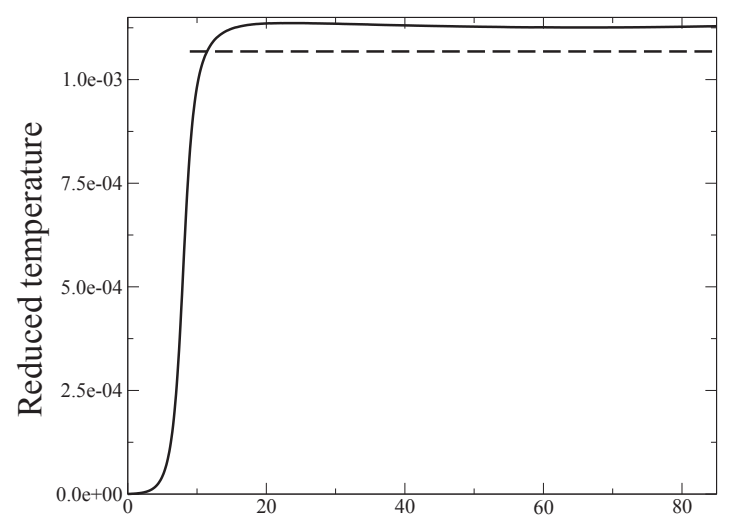

Reduced time $t^{+}=\delta_{L}^{o} t / s_{L}^{o}$

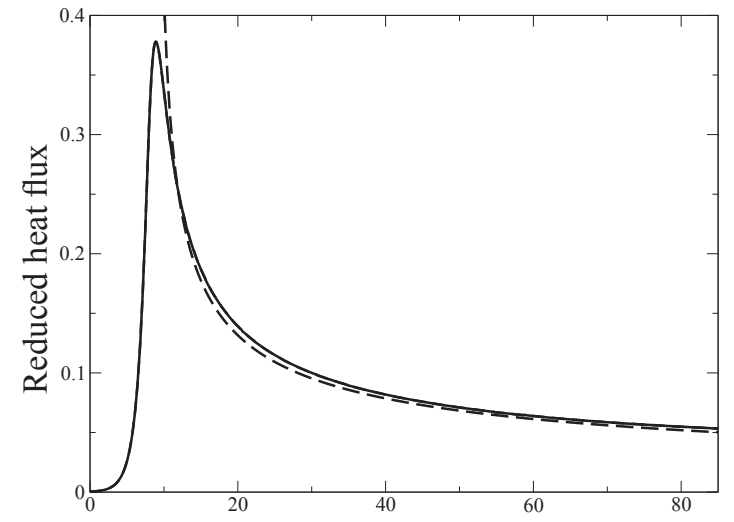

Reduced time $t^{+}=\delta_{L}^{o} t / s_{L}^{o}$

Figure 6 : Coupled flame/wall interaction simulation (solid line). Comparison with IFF limit adjusted to start at flame quenching (dashed line). Left: reduced wall temperature $(x=0)$, right: reduced wall heat flux at $x=0$. 


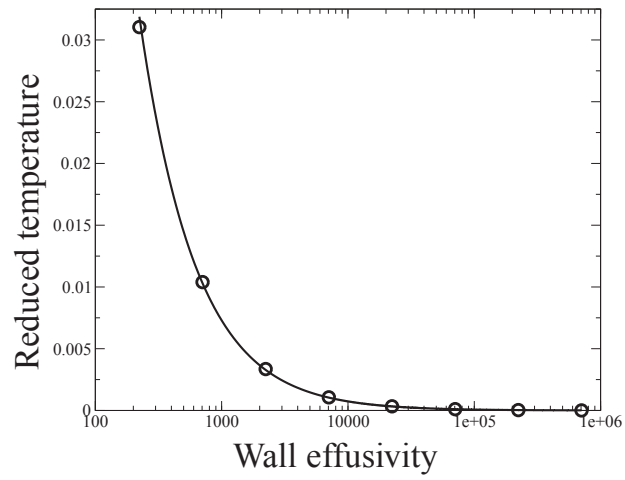

Figure 7 : Coupled flame/wall interaction simulation. Reduced wall temperature $\left(T(x=0)-T_{1}\right) /\left(T_{2}-T_{1}\right)$ vs wall effusivity $b_{s}$. Solid line: IFF limit, circles: coupled simulation.
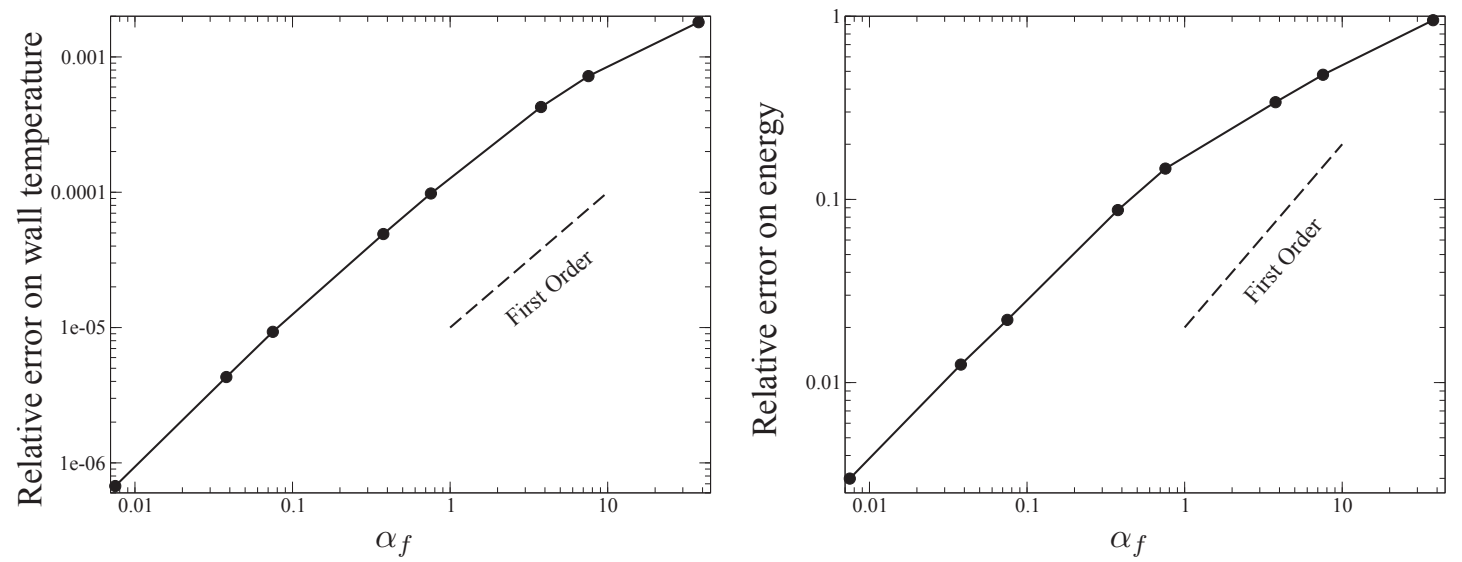

Figure 8 : Flame/wall interaction simulation. Left: relative error on wall temperature $(x=0)$ from $t^{+}=\delta_{L}^{o} t / s_{L}^{o}=0$ to $t^{+}=82$, right: relative error on the energy fluxed into the wall on the same period.

that the IFF limit given by Eq. 4 is only approximate for the flame wall interaction problem since it assumes constant density and heat diffusivity in the gases.

Finally, Fig. 8 shows how the coupling frequency (measured by the parameter $\alpha_{f}$ ) changes the precision of the coupled simulation (coupling events are scheduled at every $\alpha_{f} \tau_{f}$ times where $\tau_{f}$ is the fluid time). The precision of the coupling was checked by changing $N$ from 100 to 500000 ( $\alpha_{f}$ from $7.510^{-3}$ to 37.8 ) with $N_{i t}=10\left(\alpha_{f}=7.510^{-4}\right)$ as reference. The error on the maximum temperature remains very small even for large $\alpha_{f}$ values. The error on the maximum energy entering the wall between the initial time and an arbitrary instant (here $\delta_{L}^{o} t / s_{L}^{o}=82$ ) depends strongly on $\alpha_{f}$. As expected from results obtained for the IFF problem only (previous section), coupling the two solvers less often that $\tau_{f}\left(\alpha_{f}>1\right)$ leads to errors larger than 10 percent on the energy fluxed into the wall. The errors on temperature and energy fluxed into the wall converge both to 0 when $\alpha_{f}$ decreases with an order close to 1 .

\section{BLADE COOLING}

The second example studied during this work is the interaction between a high-speed flow and a cooled blade. This example is typical of one of the main problems encountered during the design of combustion chambers $[2,21]$ : the hot flow leaving the combustor must not burn the turbine blades or the vanes of the high pressure stator. Predicting the vanes temperature field (which are cooled from the inside by cold air) is a major research area [22, 23, 4]. Here an experimental set-up (T120D blade) developed within the AITEB2 European project was used to evaluate the precision of the coupled simulations (Fig. 9). The temperature difference between the mainstream $\left(T_{2}=333.15 K\right)$ and cooling $\left(T_{1}=303.15 K\right)$ airs is limited to $30 \mathrm{~K}$ to facilitate measurements. Experimental results include pressure data on the blade suction and pressure sides as well as temperature measurement on the pressure side.

The computational domains for both the fluid and the structure contain only one spanwise pitch of the film 


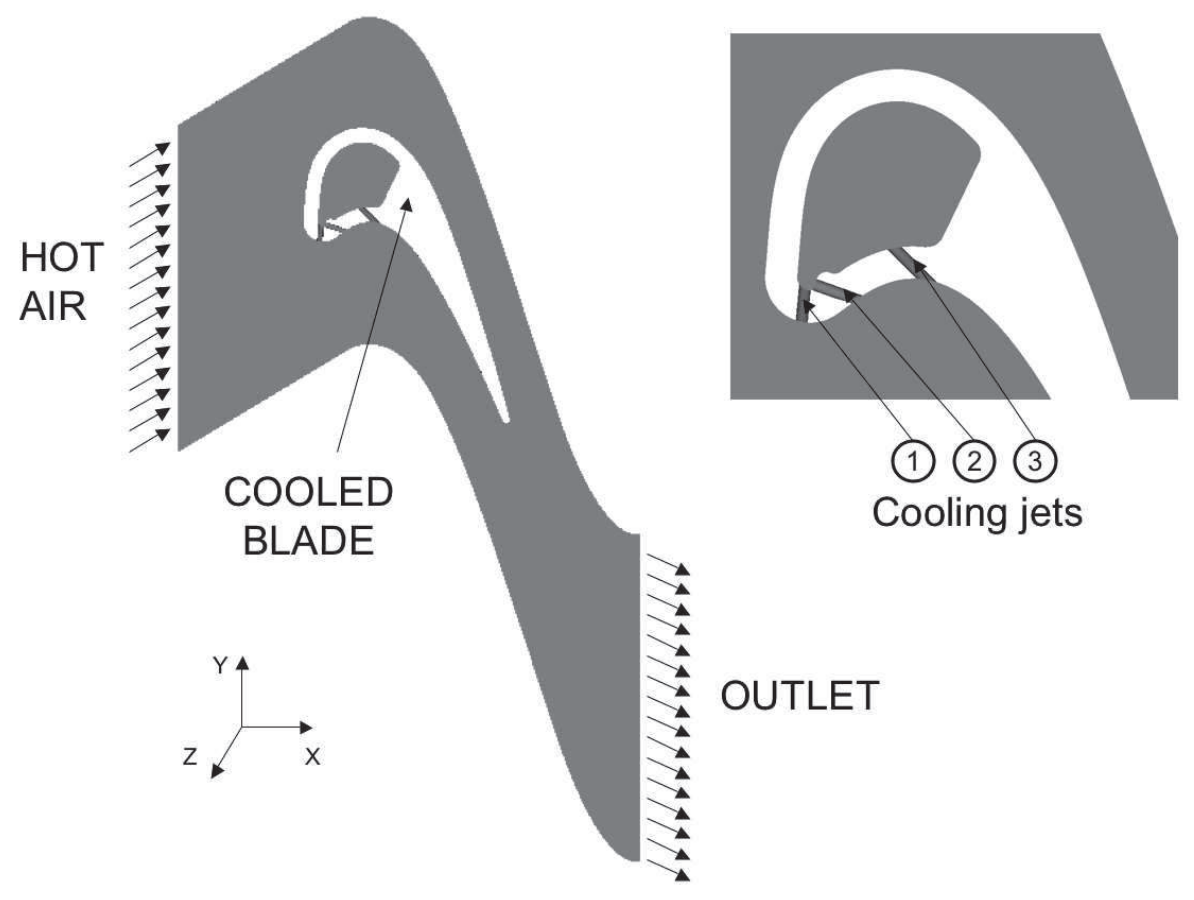

Figure 9 : Configuration for blade cooling simulation: the T120D blade (AITEB2 project).

\begin{tabular}{ccccccccc} 
& $\begin{array}{c}\text { Inlet static } \\
\text { temperature }\end{array}$ & $\begin{array}{c}\text { Inlet total } \\
\text { temperature }\end{array}$ & $\begin{array}{c}\text { Inlet total } \\
\text { pressure }\end{array}$ & $\begin{array}{c}\text { Flow } \\
\text { rate }\end{array}$ & $\begin{array}{c}\text { Thermal } \\
\text { conductivity }\end{array}$ & $\begin{array}{c}\text { Heat } \\
\text { capacity }\end{array}$ & $\begin{array}{c}\text { Time } \\
\text { scale }\end{array}$ & $\begin{array}{c}\text { Time } \\
\text { step } \Delta t_{f}^{m}\end{array}$ \\
\hline Mainstream & $T_{2}=333.15$ & $T_{2}^{t}=339.15$ & $P_{2}^{t}=27773$ & 0.0185 & $2.610^{-2}$ & 1015 & 0.001 & $9.8010^{-8}$ \\
Cooling air & $T_{1}=303.15$ & $T_{1}^{t}=303.15$ & $P_{1}^{t}=29143$ & 0.000148 & $2.4410^{-2}$ & 1015 & 0.0006 & $9.8010^{-8}$
\end{tabular}

Table 3 : Flow characteristics for the blade cooling case (SI units). The fluid time scales are based on the flow-through times in and around the blade. The characteristic fluid time scale $\tau_{f}$ is the maximum of this time, ie $\tau_{f}=0.001$. The time step $\Delta t_{f}^{m}$ is limited by the acoustic CFL number (0.7).

cooling hole pattern ( $\mathrm{z}$ axis on Fig. 9), with periodicity enforced at each end. This simplification assumes no end-wall effects, but retains the three-dimensionality of the flow and greatly reduces the number of tetrahedral cells required to model the blade: about 6.5 million cells to discretize the fluid and 600000 for the solid. A periodicity condition is also assumed in the y direction. The WALE subgrid model [24] is used in conjunction with non-slipping wall conditions. As shown on Fig. 9, the three film-cooling holes and the plenum are included in the domain: jet 2 is aligned with the main flow (in the xy plane) while jets 1 and 3 have a compound orientation. The mean blowing ratio (ratio of a jet momentum on the hot flow momentum) of the jets based on a hot gases velocity of $35 \mathrm{~ms}^{-1}$ is approximately 0.4 .

Tables 3 and 4 summarize the properties of the gases and of the solid used for the simulation. At each coupling event, fluxes and temperature on the blade skin are exchanged as described in Fig. 1. During this work, only a steady state solution within the solid was sought so that time consistency was not ensured during the coupling computation. The converged state is obtained with a two step methodology which consists in:

1. Initialization of the coupled calculation that includes:

- a thermal converged adiabatic fluid simulation,

- a thermal converged isothermal solid computation with boundary temperatures given by the fluid solution,

2. Coupled simulation.

Convergence is investigated by plotting the history of the total flux on the blade (which must go to zero) and of the mean, minimum and maximum blade temperatures. Fig. 10 and Fig. 11 show these results for two variants of the PCS strategy. In the first one, fluxes and temperature are exchanged at each coupling step 


\begin{tabular}{cccccc}
$\begin{array}{c}\text { Thermal } \\
\text { conductivity }\end{array}$ & $\begin{array}{c}\text { Heat } \\
\text { capacity }\end{array}$ & Density & $\begin{array}{c}\text { Thermal } \\
\text { diffusivity }\end{array}$ & $\begin{array}{c}\text { Time } \\
\text { scale } \tau_{s}\end{array}$ & $\begin{array}{c}\text { Time } \\
\text { step }\end{array}$ \\
\hline 0.184 & 1450 & 1190 & $1.0710^{-7}$ & 34.22 & $1.7110^{-3}$
\end{tabular}

Table 4 : Solid characteristics for the blade cooling case (SI units). The time scale $\tau_{s}$ is computed using the thermal diffusivity and the blade minimum thickness.

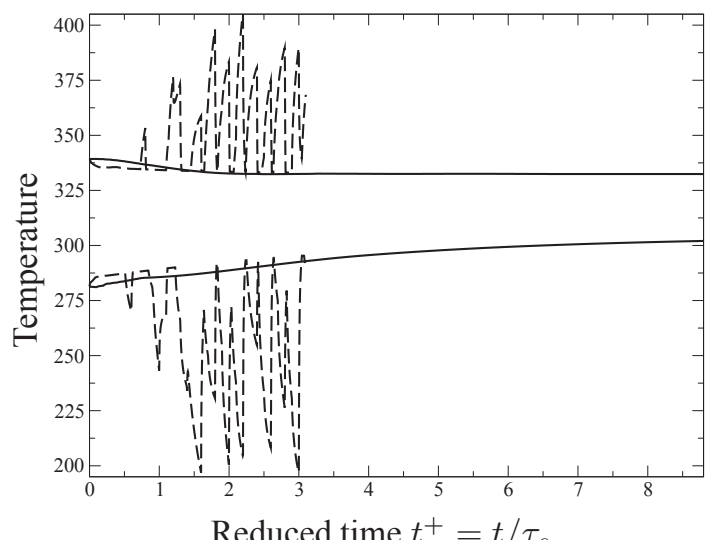

Reduced time $t^{+}=t / \tau_{s}$

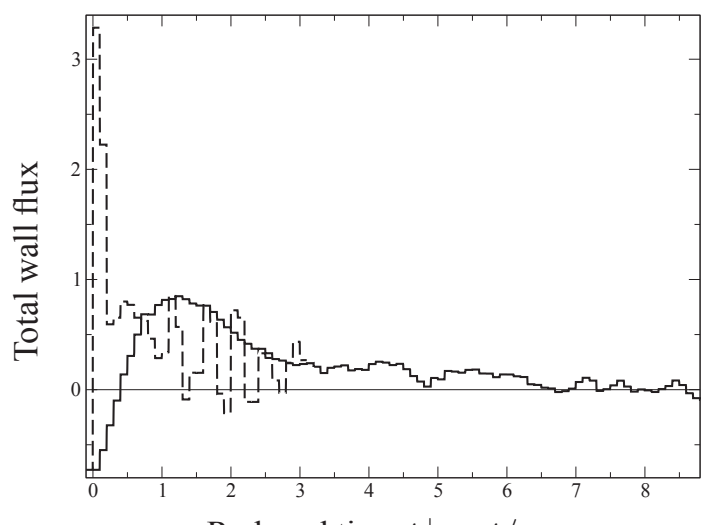

Reduced time $t^{+}=t / \tau_{s}$

Figure 10 : Time evolution of minimum and maximum temperatures in the blade (left) and total heat flux through the blade with (solid) and without (dashed) relaxation.

while for the second one, relaxation is used and temperature and fluxes imposed at each coupling iteration $n$ are written as $f^{n}=a f^{n-1}+(1-a) f^{n *}$ where $f^{n *}$ is the value obtained by the other solver at iteration $n$ and $a$ is a relaxation factor (typically $a=0.6$ ). Without relaxation, the system becomes unstable and convergence almost impossible.

At the converged state, the total flux reaches zero: the flux entering the blade is evacuated into the cooling air in the plenum and in the holes (Fig. 11). Note however that the analysis of fluxes on the blade skin shows that, even though the blade is heated by the flow on the pressure side, it is actually cooled on part of the suction side because the flow accelerates and cools down on this side. Due to the acceleration in the jets, heat transfer in the holes and plenum are of the same order. Compared to the external flux, plenum and hole fluxes converge almost linearly. Oscillations in the external flux evolution are linked with the complex flow structure developing around the blade.

At the converged state, results can be compared to the experiment in terms of pressure profiles on the blade (on both sides) and of temperature profiles on the pressure side. Pressure fields are displayed in terms of isentropic Mach numbers $M_{i s}$ computed by

$$
M_{i s}=\sqrt{\frac{2}{\gamma-1}\left[\left(\frac{P_{2}^{t}}{P_{w}^{t}}\right)^{\frac{\gamma-1}{\gamma}}-1\right]}
$$

where $P_{2}^{t}$ and $P_{w}^{t}$ are the total pressure of the main stream and at the wall. Fig. 12 displays an average field of isentropic Mach number obtained by LES and by the experiment. The comparison of the adiabatic simulation and the coupled one shows that these profiles are only weakly sensitive to the thermal condition imposed on the blade. Although the shock position on the suction side is not perfectly captured, the overall agreement between LES and experimental results is fair.

Temperature results are displayed in terms of reduced temperature $\Theta=\left(T_{2}^{t}-T\right) /\left(T_{2}^{t}-T_{1}^{t}\right)$ where $T_{2}^{t}$ and $T_{1}^{t}$ are the total temperatures of the main and cooling streams (Table 4 ) and $T$ is the local wall temperature. $\Theta$ measures the cooling efficiency of the blade. Fig. 13 shows measurements, adiabatic and coupled LES results for $\Theta$ spanwise averaged along axis x. As expected, the cooling efficiency obtained with the adiabatic computation are lower than the experimental values: the adiabatic temperature field over-predicts the real one. The main contribution of conduction in the blade is to reduce the wall temperature on the pressure side.

The reduced temperature distribution on the pressure side (Fig. 14) shows that the peak temperature occurs at the stagnation point (reduced abscissa close to 0). The temperature at the stagnation point is reduced 


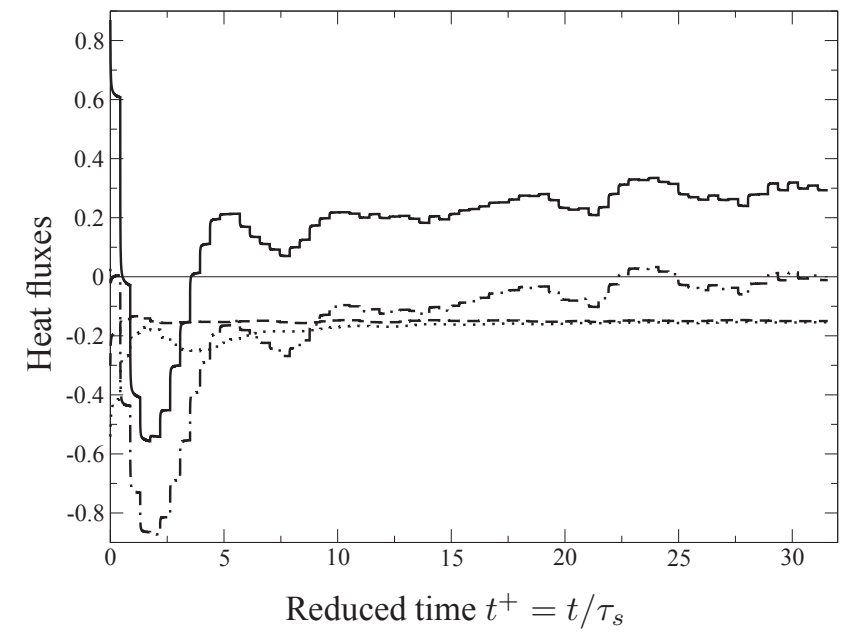

Figure 11 : Time evolution of heat fluxes through the blade: external flux (solid line),plenum (dashed), holes sides (dot), sum of all fluxes (dot dashed).

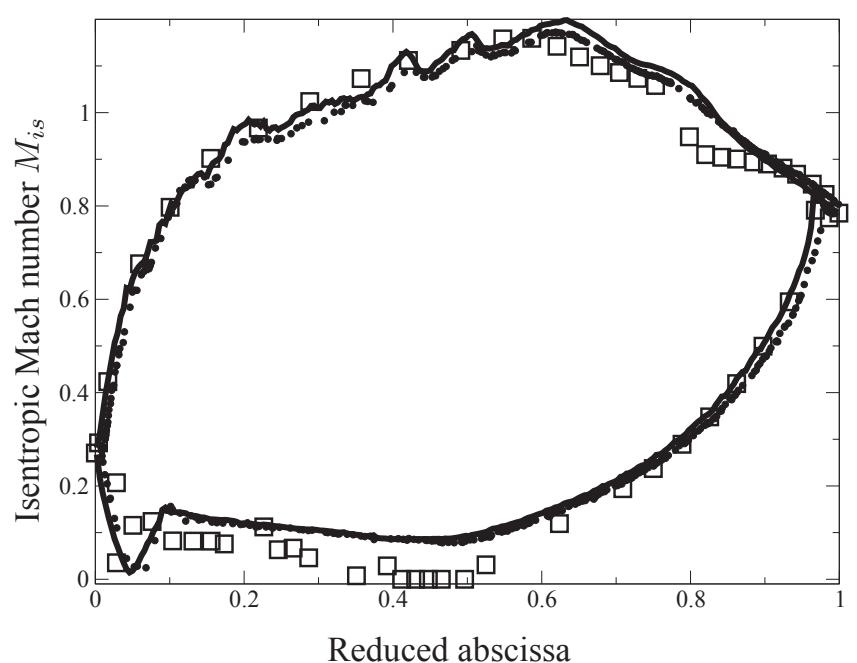

Figure 12 : Isentropic Mach number along the blade. Solid line: coupled LES, circles: adiabatic LES, squares: experiment.

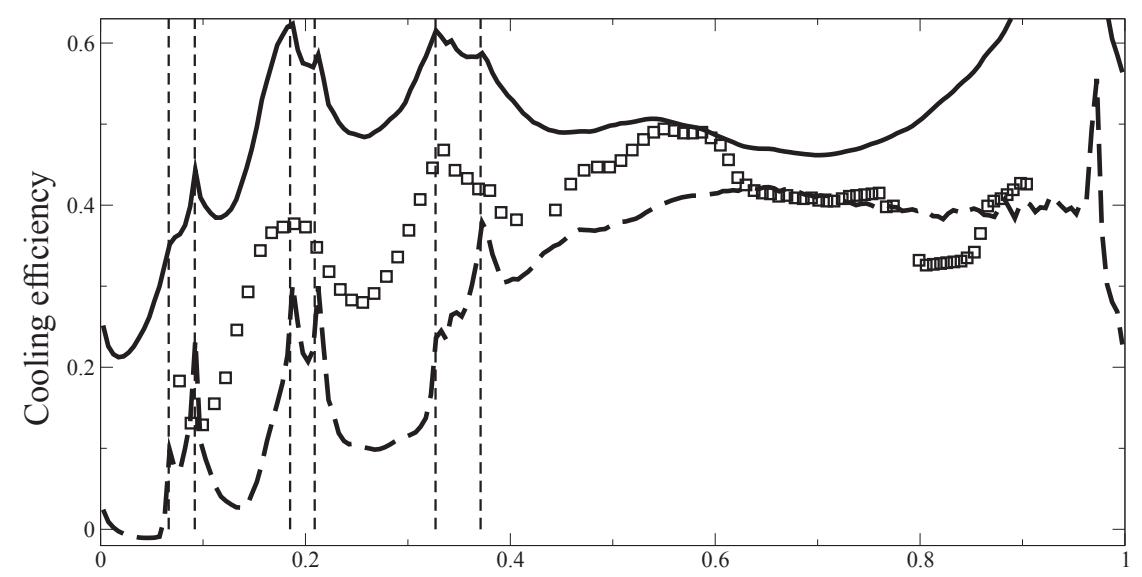

Reduced abscissa

Figure 13 : Cooling efficiency $\Theta$ versus abscissa on the pressure side at steady state. Dashed line: adiabatic LES, solid line: coupled LES, symbols: experiment, vertical dashed lines: position of the holes. 


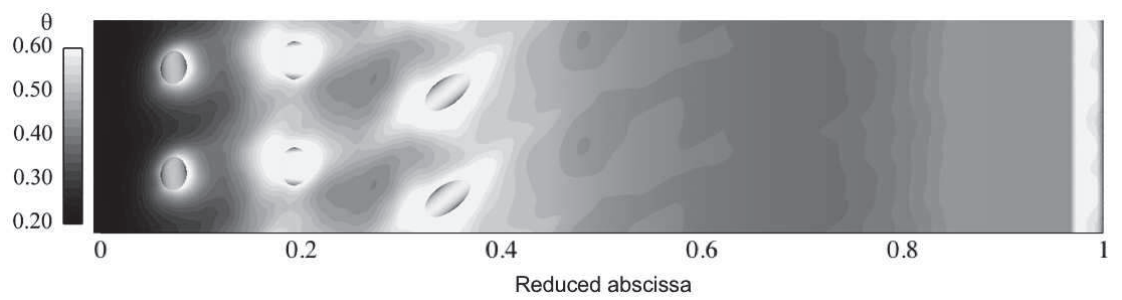

Figure 14 : Spatial distribution of reduced temperature $\theta$ on the pressure side of the blade. The computational domain is duplicated one time in the $z$ direction.

compared to the adiabatic wall prediction, leading to local values of $\theta$ of the order of 0.2 . The thermal effects of the cooling jets on the vane are clearly evidenced by Fig. 14. Jet 3 seems to be the most active in the cooling process by protecting the blade from the hot stream until a reduced absissa of 0.5 and then impacting the vane between 0.5 and 0.6 .

The reduced temperature obtained during this work over estimates experimental measurements. In particular, the strong acceleration caused by the blade induce large thermal gradients at the trailing edge. This phenomenon not well resolved by the computations leads to a non physical values of cooling efficiency. Nevertheless, these results have shown a very large sensitivity to multiple parameters, not only of the coupling strategy but also of the LES models for heat transfer and wall descriptions. Additional studies will be continued after the summer program.

\section{CONCLUSIONS}

Conjugate heat transfer calculations have been performed for two configurations of importance for the design of gas turbines with a recently developed massively parallel tool based on a LES solver. (1) An unsteady flame/wall interaction problem was used to assess the precision of coupled solutions when varying the coupling period. It was shown that the maximum coupling period that allows to well reproduce the temperature and the flux across the wall is of the order of the smallest time scale of the problem. (2) Steady convective heat transfer computation of an experimental film-cooled turbine vane showed how thermal conduction in the blade tend to reduce wall temperature compared to an adiabatic case. Further studies on LES models, coupling strategy and experimental conditions are needed to improve quality of the results compared to the experimental cooling efficiency.

The help of L. Pons from TURBOMECA and of the AITEB2 consortium to have access to the experimental results is gratefully acknowledged.

\section{REFERENCES}

[1] A. H. Lefebvre. Gas Turbines Combustion. Taylor \& Francis, 1999.

[2] R. S. Bunker. Gas turbine heat transfer: 10 remaning hot gas path challanges. In Procceedings of GT2006. ASME Turbo Expo 2006, 2006.

[3] R. Schiele and S. Wittig. Gas turbine heat transfer: Past and future challenges. Journal of Propulsion and Power, 16(4):583-589, July 2000.

[4] V.K. Garg. Heat transfer research on gas turbine airfoils at nasa grc. International Journal of Heat and Fluid Flow, 23(2):109-136, April 2002.

[5] E. Mercier, L. Tesse, and N. Savary. 3D Full Predictive Thermal Chain for Gas Turbine. In 25th International Congress of the Aeronautical Sciences, Hamburg, Germany, September 2006.

[6] T. Schönfeld and M. Rudgyard. Steady and unsteady flows simulations using the hybrid flow solver avbp. AIAA Journal, 37(11):1378-1385, 1999.

[7] P. Schmitt, T. J. Poinsot, B. Schuermans, and K. Geigle. Large-eddy simulation and experimental study of heat transfer, nitric oxide emissions and combustion instability in a swirled turbulent high pressure burner. J. Fluid Mech., 570:17-46, 2007. 
[8] O. Colin and M. Rudgyard. Development of high-order taylor-galerkin schemes for unsteady calculations. J. Comput. Phys., 162(2):338-371, 2000.

[9] V. Moureau, G. Lartigue, Y. Sommerer, C. Angelberger, O. Colin, and T. Poinsot. High-order methods for DNS and LES of compressible multi-component reacting flows on fixed and moving grids. J. Comput. Phys., 202(2):710-736, 2005.

[10] T. Poinsot and D. Veynante. Theoretical and numerical combustion. R.T. Edwards, 2nd edition., 2005.

[11] S. Buis, A. Piacentini, and D. Déclat. PALM: A Computational Framework for assembling High Performance Computing Applications. CONCURRENCY AND COMPUTATION: PRACTICE AND EXPERIENCE, 2005.

[12] M.B. Giles. Stability analysis of numerical interface conditions in fluid-structure thermal analysis. International Journal for Numerical Methods in Fluids, 25(4):421-436, 1997.

[13] S. Chemin. Étude des Interactions Thermiques Fluides-Structure par un Couplage de Codes de Calcul. PhD thesis, Université de Reims Champagne-Ardenne, 2006.

[14] A. Delataillade, F. Dabireau, B. Cuenot, and T. Poinsot. Flame/wall interaction and maximum heat wall fluxes in diffusion burners. Proc. Combust. Inst., 29:775-780, 2002.

[15] F. Dabireau, B. Cuenot, O. Vermorel, and T. Poinsot. Interaction of h2/o2 flames with inert walls. Combust. Flame, 135(1-2):123-133, 2003.

[16] J. H. Lu, O. Ezekoye, R. Greif, and F. Sawyer. Unsteady heat transfer during side wall quenching of a laminar flame. In 23rd Symp. (Int.) on Combustion, pages 441-446. The Combustion Institute, Pittsburgh, 1990.

[17] O. A. Ezekoye, R. Greif, and D. Lee. Increased surface temperature effects on wall heat transfer during unsteady flame quenching. In 24th Symp. (Int.) on Combustion, pages 1465-1472. The Combustion Institute, Pittsburgh, 1992.

[18] G. Desoutter, B. Cuenot, C. Habchi, and T. Poinsot. Interaction of a premixed flame with a liquid fuel film on a wall. Proc. Combust. Inst., 30:259-267, 2005.

[19] P. Popp, M. Baum, M. Hilka, and T. Poinsot. A numerical study of laminar flame wall interaction with detailed chemistry: wall temperature effects. In T. J. Poinsot, T. Baritaud, and M. Baum, editors, Rapport du Centre de Recherche sur la Combustion Turbulente, pages 81-123. Technip, Rueil Malmaison, 1996.

[20] P. Popp and M. Baum. An analysis of wall heat fluxes, reaction mechanisms and unburnt hydrocarbons during the head-on quenching of a laminar methane flame. Combust. Flame, 108(3):327-348, 1997.

[21] D. G. Bogard and K. A. Thole. Gas turbine film cooling. J. Prop. Power, 22(2):249-270, 2006.

[22] M.-L. Holmer, L.-E. Eriksson, and B. Sunden. Heat transfer on a film cooled inlet guide vane. In Proceedings of the ASME Heat Transfer Division, volume 366-3, pages 43-50, 2000.

[23] G. Medic and P. A. Durbin. Toward improved film cooling prediction. J. Turbomach., 124:193-199, 2002.

[24] F. Nicoud and T. Poinsot. Dns of a channel flow with variable properties. In Int. Symp. On Turbulence and Shear Flow Phenomena., Santa Barbara, Sept 12-15., 1999. 